\title{
Full Characterization of in vivo Muscle as an Elastic, Incompressible, Transversely Isotropic Material using Ultrasonic Rotational 3D Shear Wave Elasticity Imaging
}

\author{
Anna E. Knight, Student Member, IEEE, Courtney A. Trutna, Student Member, IEEE, Ned C. Rouze, \\ Member, IEEE, Lisa D. Hobson-Webb, Annette Caenen, Member, IEEE, Felix Q. Jin Student Member, \\ IEEE, Mark L. Palmeri, Member, IEEE, Kathryn R. Nightingale, Senior Member, IEEE
}

\begin{abstract}
Using a 3D rotational shear wave elasticity imaging (SWEI) setup, 3D shear wave data were acquired in the vastus lateralis of a healthy volunteer. The innate tilt between the transducer face and the muscle fibers results in the excitation of multiple shear wave modes, allowing for more complete characterization of muscle as an elastic, incompressible, transversely isotropic (ITI) material. The ability to measure both the shear vertical (SV) and shear horizontal (SH) wave speed allows for measurement of three independent parameters needed for full ITI material characterization: the longitudinal shear modulus $\mu_{\mathbf{L}}$, the transverse shear modulus $\mu_{\mathrm{T}}$, and the tensile anisotropy $\chi_{\mathbf{E}}$. Herein we develop and validate methodology to estimate these parameters and measure them in vivo, with $\mu_{\mathbf{L}}=5.77 \pm 1.00 \mathrm{kPa}, \mu_{\mathbf{T}}=1.93 \pm 0.41 \mathrm{kPa}$ (giving shear anisotropy $\chi_{\mu}=2.11 \pm 0.92$ ), and $\chi_{\mathbf{E}}=4.67 \pm 1.40$ in a relaxed vastus lateralis muscle. We also demonstrate that 3D SWEI can be used to more accurately characterize muscle mechanical properties as compared to 2D SWEI.
\end{abstract}

Index Terms-Muscle, Shear Wave Elastography, Transverse Isotropy

\section{INTRODUCTION}

$\mathbf{S}$ HEAR wave elasticity imaging (SWEI) and other acoustic radiation force impulse (ARFI) methods have been used to measure mechanical properties in a wide variety of tissues [1][3]. ARFI and SWEI methods employ a conventional clinical ultrasound transducer to create a small amplitude $(<50 \mu \mathrm{m})$ localized displacement in the tissue, inducing shear waves that spread outward radially from the push location and are tracked

A. Knight, C. Trutna, N. Rouze, F. Jin, M. Palmeri, and K. Nightingale are with the Department of Biomedical Engineering, Duke University, Durham, NC, 27707 USA e-mail: anna.knight@duke.edu

L. Hobson-Webb is with Duke Department of Neurology, Duke University Health System, Durham, NC, 27707, USA

A. Caenen is with Ghent University, Department of Electronics and Information Systems, IBiTech-bioMMeda Lab Ghent, BELGIUM and Cardiovascular Sciences Department, Cardiovascular Imaging and Dynamics Lab at KU Leuven, Leuven, BELGIUM

The authors would like to thank the funding sources: NIH grant R01CA142824, R01EB022106, and the Duke MEDx Pilot Project Grant.

K. Nightingale, N. Rouze and M. Palmeri hold intellectual property related to acoustic radiation force impulse and shear wave elasticity imaging.

Manuscript received Dec, 4 2020; revised December 30, 2020. by subsequent ultrasound frames [4]. Under the assumption of a linear, isotropic, and elastic material [4]-[6], the shear modulus $\mu$ can be calculated from the measured shear wave speed $c$ and tissue density $\rho$ as

$$
\mu=\rho c^{2} .
$$

Musculoskeletal SWEI is a wide field of research. Studies have indicated differences in shear wave speeds related to sex [7]-[9], age [8], [10], [11], contractile state [12]-[14] and muscle related diseases such as myopathies [15], [16], dystrophies [17], [18], and muscle spasticities [19]-[22]. The existing research shows uncertainty and lack of repeatability among SWEI measurements in skeletal muscle using current tools [23]-[28], which limits clinical adoption. However, the volume of research to date demonstrates an interest by the orthopedic and neurology communities in noninvasively assessing muscle material properties.

Skeletal muscle is commonly modeled as a transversely isotropic (TI) material due to the alignment of muscle fibers along an axis of symmetry, typically oriented along the direction of muscle contraction. A linear, elastic TI material can be characterized by five parameters [29]-[34]. Furthermore, if the material is assumed to be incompressible, these five parameters are reduced to three [29], [30]. In this study we model skeletal muscle as an incompressible TI (ITI) material characterized by three parameters: the longitudinal shear modulus $\mu_{L}$, the transverse shear modulus $\mu_{T}$, and a single parameter modeling the joint effect of the longitudinal and transverse Young's moduli $E_{L}$ and $E_{T}$. In previous work we have reported this third parameter as the ratio $E_{T} / E_{L}$ [29], [30]; herein we report tensile anisotropy $\chi_{E}$ [35], defined as

$$
\chi_{E}=\frac{E_{L}-E_{T}}{E_{T}}=\frac{1}{E_{T} / E_{L}}-1 .
$$

This allows for easy comparison to shear anisotropy $\chi_{\mu}$ [35], defined as

$$
\chi_{\mu}=\frac{\mu_{L}-\mu_{T}}{\mu_{T}} .
$$

Many studies to date have measured the directional dependence of shear wave propagation in muscle, reporting a wide 

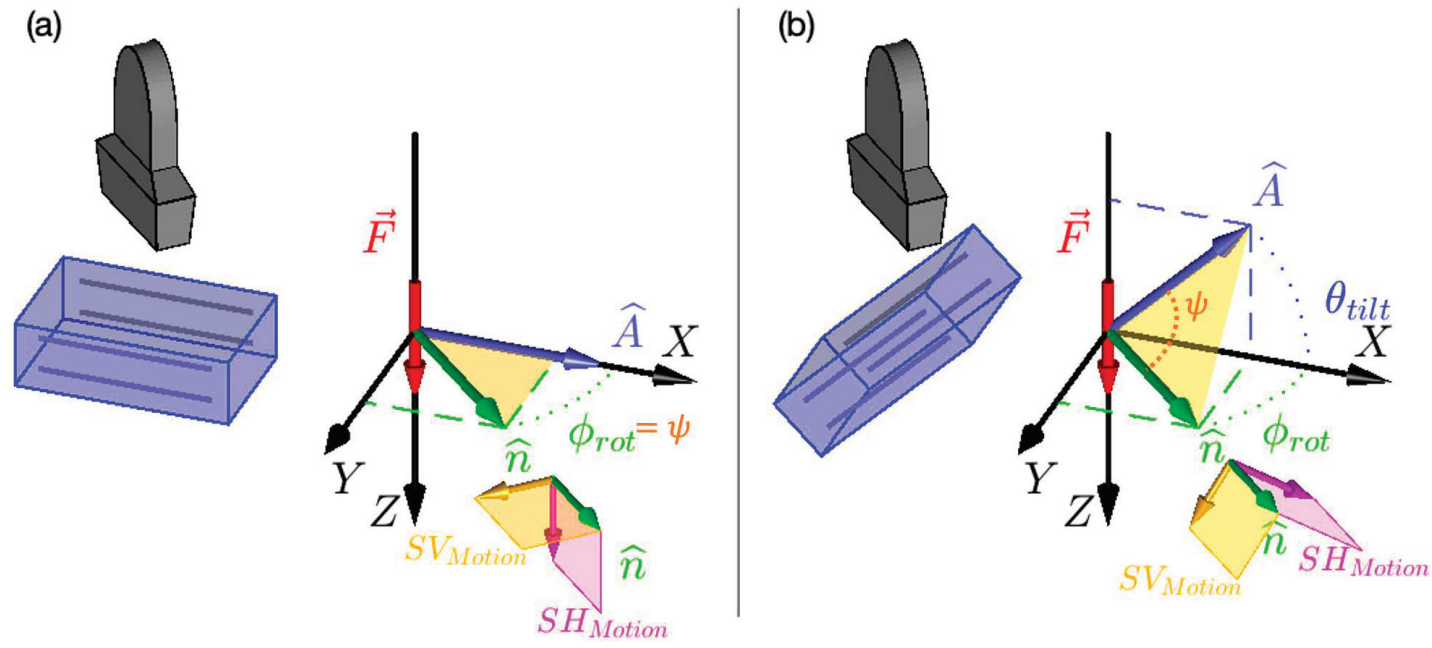

Fig. 1. (a) 3D SWEI orientation with ARFI push excitation $\widehat{\boldsymbol{F}}$ normal to muscle fiber direction (i.e. muscle symmetry axis, $\widehat{\boldsymbol{A}}$ ). $\widehat{\boldsymbol{n}}$ represents the direction of wave propagation along which the shear wave speed is computed. $\phi_{\text {rot }}$ is the angle between $\widehat{A}$ and $\widehat{\boldsymbol{n}}$ in the $\boldsymbol{X} \boldsymbol{Y}$ plane. In a TI material, two shear wave modes with different polarizations can be excited: the shear horizontal (SH) mode, shown in pink, which is always perpendicular to the $\widehat{\boldsymbol{A}}-\widehat{\boldsymbol{n}}$ plane and the shear vertical (SV) mode, shown in yellow, which is in the $\widehat{\boldsymbol{A}}-\widehat{\boldsymbol{n}}$ plane. With $\widehat{\boldsymbol{F}}$ and $\widehat{\boldsymbol{A}}$ at normal incidence, as in (a), the $\mathrm{SH}$ mode is the primary mode excited in this configuration. (b) 3D SWEI orientation with ARFI push excitation $\widehat{\boldsymbol{F}}$ at an angle $\boldsymbol{\theta}_{\text {tilt }}$ away from normal incidence to the muscle symmetry axis $(\widehat{A})$. With a nonzero $\boldsymbol{\theta}_{\text {tilt }}$, both the SH and SV modes are excited and have displacement components in the $Z$ (axial) imaging direction in the material. The angle $\boldsymbol{\psi}$ between $\widehat{\boldsymbol{A}}$ and $\widehat{\boldsymbol{n}}$ is shown in orange in (b), while in (a) $\psi=\phi_{\text {rot }}$.

range of speeds along and across the fiber axis [16], [24], [36][50]. These reports used an experimental setup similar to that shown in Fig. 1(a), where the fiber symmetry axis is in a plane parallel to the transducer face, allowing for the measurement of shear wave speeds along and across the muscle fiber axis. The shear moduli $\mu_{L}$ and $\mu_{T}$ that characterize shear wave propagation along and across the muscle fiber axis can be calculated from these speeds using (1). However tensile anisotropy $\chi_{E}$ cannot be determined using (1).

Recently, our group has demonstrated that it is possible to excite and observe multiple shear wave propagation modes in a uniaxially stretched polyvinyl alcohol phantom in which the stretch axis represents the material symmetry axis $\widehat{A}$ [51]. This experimental setup is similar to that shown in Fig. 1(b). The key difference compared to the typical setup shown in Fig. 1(a) is that the material symmetry axis $\widehat{A}$ is tilted with respect to the ultrasonic acoustic radiation force push beam $\widehat{F}$ $(\widehat{A} \not \perp \widehat{F})$. With this setup, both the SH and SV propagation modes are excited by an ARFI force directed along the $\mathrm{Z}$ axis. Measurement of both the SH and SV wave modes enables characterization not only of $\mu_{L}, \mu_{T}$, and $\chi_{\mu}$ from the $\mathrm{SH}$ mode, as measured by a typical setup (Fig. 1(a)), but also of $\chi_{E}$, which is dependent upon the SV mode. Herein, we present a method to exploit this geometry and estimate $\mu_{L}, \mu_{T}, \chi_{\mu}$ and $\chi_{E}$ using Green's function (GF) simulations. We validated our method using finite element method (FEM) simulations, and we also apply this approach to estimate these parameters in in vivo muscle.

\section{BACKGROUND}

\section{A. Linear, Elastic, Incompressible, Transversely Isotropic} (ITI) Material Models

A linear, elastic material's stress-strain relationship can be written in terms of a generalized Hooke's law between the stress tensor $\sigma$ and strain tensor $\epsilon$ as

$$
\sigma_{i j}=c_{i j k l} \epsilon_{k l}
$$

where $c_{i j k l}$ are the elements of the stiffness tensor that characterize the material. For a TI material, rotational and reflective symmetries of the material about its symmetry axis $\widehat{A}$ (Fig. 1) reduce the stiffness tensor to five independent quantities [30]-[33]. These five quantities can be expressed in terms of six engineering constants and one constraint: two Young's moduli $E_{L}$ and $E_{T}$ along the longitudinal (L) and transverse (T) directions, two shear moduli $\mu_{L}$ and $\mu_{T}$, two Poisson's ratios, $\nu_{L T}$ and $\nu_{T T}$, and the constraint

$$
\mu_{T}=\frac{E_{T}}{2\left(1+\nu_{T T}\right)} .
$$

In SWEI methods, soft tissues are often treated as quasiincompressible materials with an acoustic wave (compressional) speed of $1540 \mathrm{~m} / \mathrm{s}$, and the shear wave propagation is modeled using incompressible material models. For the case of an ITI material, the Poisson's ratios, $\nu_{L T}$ and $\nu_{T T}$ have specific values [29], [30]

$$
\nu_{L T}=1 / 2
$$

and

$$
\nu_{T T}=1-\frac{1}{2} \frac{E_{T}}{E_{L}} .
$$

These relations, plus the constraint in equation (5), imply that shear wave propagation in an ITI material can be described by three independent material parameters. Here, we use the parameters $\mu_{T}, \mu_{L}$, and $\chi_{E}$. Note that we have previously used $E_{T} / E_{L}$ [29], [30] which is related to $\chi_{E}$ by (2). 


\section{B. Wave Propagation in ITI Materials}

Plane wave propagation in a TI material can be described in terms of three propagation modes: the pressure or compressional mode $(\mathrm{P})$, in which the polarization is aligned with the propagation direction $\widehat{n}$ (Fig. 1), and the shear horizontal (SH) and shear vertical (SV) wave modes, in which the polarization is perpendicular to the propagation direction $\widehat{n}$. Particle motion in the $\mathrm{SH}$ wave mode is in the plane perpendicular to the plane formed by the material symmetry axis $\widehat{A}$ and propagation direction $\widehat{n}$ (the pink planes in Fig. 1), while particle motion in the SV wave mode is in the $\widehat{A}-\widehat{n}$ plane (yellow planes in Fig. 1).

In the limit of an ITI material, the phase velocity of the $\mathrm{P}$-wave becomes infinite, and we will ignore this propagation mode. The phase velocities $v_{S H}$ and $v_{S V}$ of the SH and SV modes are given in terms of the angle $\psi$ between $\widehat{A}$ and $\widehat{n}$ [29], [30] as

$$
\rho v_{S H}^{2}=\mu_{L} \cos ^{2} \psi+\mu_{T} \sin ^{2} \psi
$$

and

$$
\rho v_{S V}^{2}=\mu_{L}+4\left(\left(\chi_{E}+1\right) \mu_{T}-\mu_{L}\right) \sin ^{2} \psi \cos ^{2} \psi
$$

Equation (8) shows that the SH mode depends only on the observation direction, $\mu_{L}$, and $\mu_{T}$, but is not affected by the tensile anisotropy, $\chi_{E}$. For this mode, the group velocity $V_{S H}(\psi)$ is given by an elliptical shape [30], [32], [34], [52] as

$$
\rho V_{S H}^{2}(\psi)=\frac{\mu_{L} \mu_{T}}{\mu_{L} \sin ^{2} \psi+\mu_{T} \cos ^{2} \psi} .
$$

The group velocity for the SV propagation mode does not reduce to a simple expression like (10) as the SV mode can support multiple shear wave speeds for a particular propagation direction, depending on the specific material parameters considered (see Fig. 1 of Rouze et al. [29]). However, we have recently reported tractable calculations using Green's functions that describe both the SV and SH modes in an elastic, ITI material, which we employ herein to characterize $\chi_{E}$ [30].

\section{Practical Implementation in Ultrasound}

In each of the two configurations seen in Fig. 1, the transducer rotates around its central axis. As such, each 2D imaging frame contains the axial imaging dimension $\mathrm{Z}$, perpendicular to the transducer face, and a lateral (radial) dimension, parallel to the transducer face, corresponding to $\widehat{n}$ in Fig. 1 as determined by the rotational angle $\phi_{\text {rot }}$ of the transducer.

SWEI methods preferentially push and track material displacements in the axial $(\mathrm{Z})$ dimension. As such, when the excitation $\widehat{F}$ from the transducer is normal to the fiber direction $\widehat{A}$ (Fig. 1(a), $\widehat{F} \perp \widehat{A}$ ), we only track the SH mode of propagation. In this configuration, the SV mode does not have an appreciable component in the $\mathrm{Z}$ (axial imaging) direction, and thus would not be detected with our experimental setup. However when the fiber direction $\widehat{A}$ is tilted relative to the transducer face, as seen in Fig. 1(b), the planes containing the SV mode and SH modes are tilted such that both modes have components in the $\mathrm{Z}$ dimension, and thus can be excited and monitored with our experimental setup. In both Figs. 1(a) and (b) the transducer rotates around its axis to allow us to measure the shear wave propagation in 3D (see Supplemental Animation 1). Similarly, the tilt angle $\theta_{\text {tilt }}$ between the fibers $\widehat{A}$ and the lateral imaging axis at each propagation direction $\widehat{n}$ can change depending on the probe positioning $\left(\phi_{\text {rot }}\right)$ and muscle architecture because different muscles have different fiber orientations relative to the skin surface. This affects the magnitude of the tissue displacement in the axial imaging orientation (Z) for both the SV and SH modes (see Supplemental Animation 2).

Typically, experiments using 2D SWEI determine $\mu_{L}$ and $\mu_{T}$ from measurements of the group shear wave speed (SWS) along and across the muscle fibers using the configuration shown in Fig. 1(a). Because the tensile anisotropy $\chi_{E}$ appears only in the equation describing the SV wave mode (9), it is necessary to measure both the $\mathrm{SV}$ and $\mathrm{SH}$ waves to fully characterize an ITI material. This is achieved herein by leveraging the tilted fiber geometry inherent to the vastus lateralis during in vivo imaging.

\section{Methods}

\section{A. Experimental 3D SWEI in in vivo Muscle}

A volumetric rotational 3D SWEI acquisition system was developed using a rotating linear array probe and a standard SWEI sequence [51], [53]. A Philips ATL L7-4 array (Philips ${ }^{\circledR}$, Andover, MA) was attached to a Verasonics Vantage 256 (Verasonics ${ }^{\circledR}$, Kirkland, WA) ultrasound system. The transducer was mounted in a custom holder attached to a rotation stage (XPS-Q8, Newport $\AA$, Irvine, CA) to allow rotation about the transducer's vertical axis (Fig. 2(a)).

A healthy, 53 year old female volunteer with no known muscular diseases or injuries was selected and written informed consent was obtained for participation in a Duke University Institutional Review Board-approved study. The subject was placed in a lateral position with a rolled towel between the knees. A water bath $(300 \mathrm{~mL})$ with Tegaderm bottom was used to ensure a consistent water path between the transducer and the muscle throughout the full rotation of the probe. Ultrasound gel was applied between the leg and the Tegaderm box, and any air bubbles were manually removed by smoothing the Tegaderm face against the leg. The water bath and transducer were positioned so the center of rotation was in the main body of the vastus lateralis muscle, approximately halfway between the greater trochanter and the quadriceps tendon (Fig. 2(a)).

Thirty-six frames were acquired in 5 degree transducer rotation $\phi_{\text {rot }}$ increments with a total of 3 seconds between each frame, including time for the transducer to rotate. As each frame included a left moving and right moving wave, the transducer only had to be rotated 180 degrees to acquire a full 360 degree volume. The sequence for each rotation position consisted of a $4 \mathrm{MHz}$ push focused at $20 \mathrm{~mm}$ axially, centered under the transducer laterally. Elevated acoustic output was employeed for the acoustic radiation force push with an $\mathrm{MI}=$ 

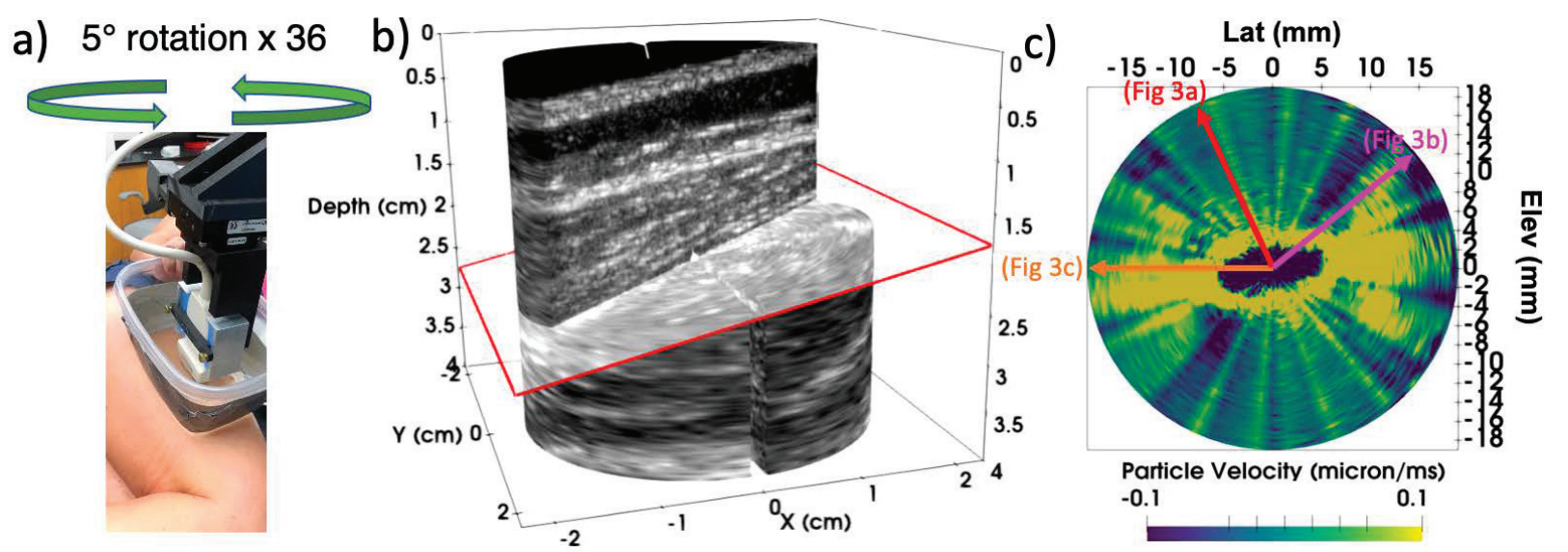

Fig. 2. Data acquisition setup. (a) shows an L7-4 transducer in the rotation stage set up (b) shows an example 3D B-mode image with a cutout at the rotational imaging planes at $\phi_{\text {rot }}=15^{\circ}$. (c) shows an example view of the particle velocity shear wave signal in the $X Y$ plane $(D e p t h=20$ $\mathrm{mm}$ ) plane at a time of $3 \mathrm{~ms}$. The colored arrows indicate transducer rotation angle $\phi_{\text {rot }}$ corresponding to the selected space time plots in Fig. 3

\begin{tabular}{|l|l|l|l|}
\hline \multicolumn{2}{|c|}{ Push } & \multicolumn{2}{c|}{ Track } \\
\hline Freq & $4.0 \mathrm{MHz}$ & Freq & $5.2 \mathrm{MHz}$ \\
\hline Focus & $20 \mathrm{~mm}$ & PRF & $10 \mathrm{kHz}$ \\
\hline$F \#$ & 0.52 & Plane Wave Angles & $-3^{\circ}, 0^{\circ}, 3^{\circ}$ \\
\hline Pulse Duration & $150 \mu \mathrm{s}$ & Acquisition Time & $40 \mathrm{~ms}$ \\
\hline MI & 3.9 & Axial Pixel Resolution & $400 \mu \mathrm{m}$ \\
\hline$I_{\text {sppa }, \alpha=0.8}$ & $1739 \mathrm{~W} / \mathrm{cm}^{2}$ & Lateral Pixel Resolution & $200 \mu \mathrm{m}$ \\
\hline$I_{\text {spta }, \alpha=0.8}$ & $91 \mathrm{~mW} / \mathrm{cm}^{2}$ & \multicolumn{2}{|l}{} \\
\cline { 1 - 2 } & \multicolumn{2}{|l}{}
\end{tabular}

TABLE I

SELECTED PUSH AND TRACK SEQUENCE PARAMETERS

3.9 and an $I_{\text {sppa, } \alpha=0.8}=1739 \mathrm{~W} / \mathrm{cm}^{2}$, and a pulse duration of $150 \mu \mathrm{s}$, and an $I_{\text {spta }, \alpha=0.8}=91 \mathrm{~mW} / \mathrm{cm}^{2}$ [54]. Each push was followed by a repeating pattern of $5.2 \mathrm{MHz}$ tracking plane waves at $10 \mathrm{kHz}$ pulse repetition frequency angled at $-3^{\circ}, 0^{\circ}$, and $3^{\circ}$ consecutively for a total acquisition time of $40 \mathrm{~ms}$. The tracking planes were coherently compounded with a rolling window, to create a $4 \times 4 \mathrm{~cm}($ axial $\times$ lateral) imaging plane region of interest with a a pixel resolution of $400 \mu \mathrm{m}$ axially and $200 \mu \mathrm{m}$ laterally in each plane. A table summarizing imaging parameters can be seen in Table I. This process of acquiring a full 360 degree rotation acquisition (36 frames) was performed a total of 4 times on two different days in the same subject and same leg.

Within each rotational plane, axial velocity data were calculated using progressive referencing and Kasai's algorithm with a $6 \lambda$ kernel [55], and then separated into the left and right moving shear waves. The velocity data were temporally low pass filtered at $600 \mathrm{~Hz}$. The transverse plane (X-Y imaging plane in Fig. 1) was extracted for further analysis by averaging over a $1 \mathrm{~mm}$ depth axially centered around the push focal depth of $20 \mathrm{~mm}$. A sample B-mode volume is shown in Fig. 2(b) with transducer rotation position $\phi_{\text {rot }}=15^{\circ}$ in the cutout plane and shows the tilted fiber orientation in the lateralis muscle. The fiber tilt angle $\theta_{t i l t}=11.4^{\circ}$ was determined by 2DFT of the B-mode image, as described in Section III-C.

\section{B. Shear Wave Speed Estimation}

Shear wave speeds were calculated for each space-time trajectory using the Radon sum algorithm [56]. Typically, a lateral range of 8 to $16 \mathrm{~mm}$ was used in the estimate, however, this range was adjusted for each space-time trajectory based on the range over which a distinct trajectory could be visually identified. The white lines overlaid on the space time signals in Fig. 3 show the lateral range used in the speed estimate. If a single wave mode was present, it was assumed to be the SH mode (Fig. 3(a)); if two trajectories were present (Fig. 3(b)), the faster was assumed to be the SV mode. In this case, individual speeds were estimated by masking the Radon sum images (see Fig. 2 of Rouze et al. [56]) to identify the trajectory for each propagation mode. All trajectories were verified visually by a single reader.

For some trajectories, such as Fig. 3(c) it was not possible to assign the estimated speed to either the SH or SV mode as the waves appeared merged. In these cases the speeds from these propagation directions were omitted from further analysis.

\section{Estimating $\widehat{A}, \theta_{\text {tilt }}, \phi_{\text {fiber }}, \mu_{\mathrm{L}}$, and $\mu_{\mathrm{T}}$}

A flowchart explaining the ITI parameter estimation algorithm is provided in Fig. 4. The two angles that specify the orientation of the muscle fibers $(\widehat{A})$ with respect to the XYZ coordinate system are rotation angle $\phi_{\text {fiber }}$, defined as the rotation angle of the projection of $\widehat{A}$ in the $X-Y$ plane, and the fiber tilt angle $\theta_{\text {tilt }}$.

To determine $\widehat{A}$, preliminary estimates assuming no fiber tilt, $\theta_{\text {tilt }}=0$, as indicated by superscript $p$ of $\mu_{L}^{p}$ and $\mu_{T}^{p}$ were found by assuming an elliptical shape for the SH mode shear wave speeds in the $X Y$ plane based on (10),

$$
V(\Delta \phi)=\left(\frac{1}{\rho} \frac{\mu_{L}^{p} \mu_{T}^{p}}{\mu_{L}^{p} \sin ^{2}(\Delta \phi)+\mu_{T}^{p} \cos ^{2}(\Delta \phi)}\right)^{1 / 2}
$$

where $\Delta \phi=\phi_{\text {rot }}-\phi_{\text {fiber }}$. Nonlinear least squares fitting was used to estimate $\mu_{L}^{p}, \mu_{T}^{p}$, and $\phi_{\text {fiber }}$ by varying these parameters and choosing the values that minimize the sum of 

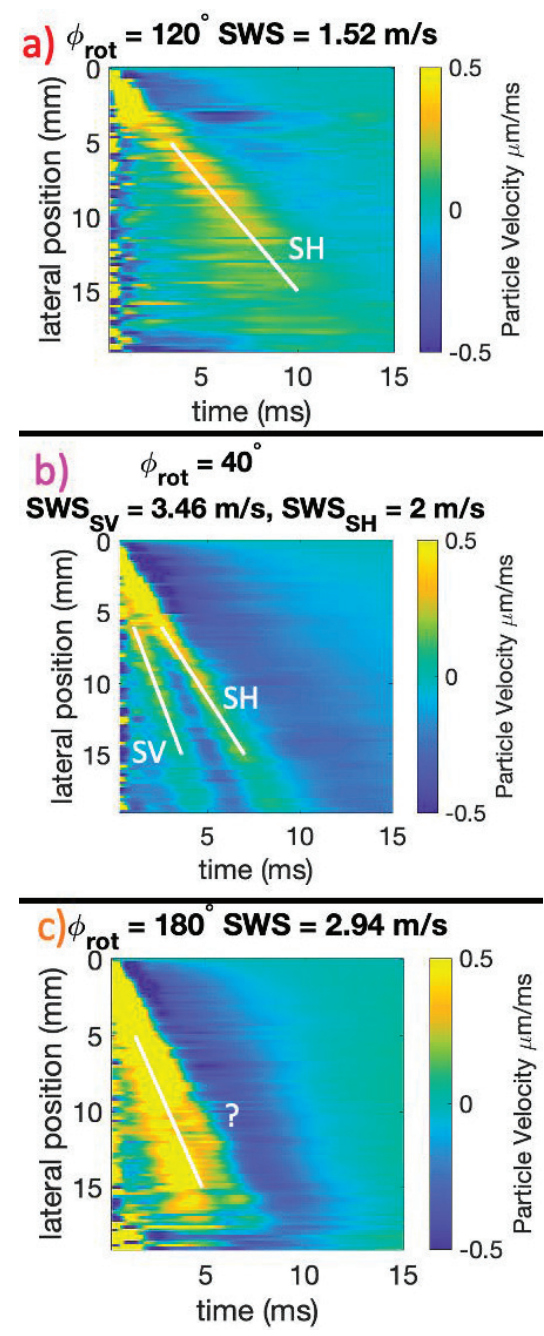

Fig. 3. Example space time plots from a single 3D experimental acquisitions. (a) shows an example space time plot of a transducer angle $\left(\phi_{\text {rot }}=\mathbf{1 2 0}^{\circ}\right)$ where there is only one trajectory and one wave mode present, which is the $\mathrm{SH}$ mode. (b) shows an example of an angle $\left(40^{\circ}\right)$ where there are two shear waves clearly present on the space time plot. The faster wave is the SV mode $(3.49 \mathrm{~m} / \mathrm{s})$ and the slower the SH mode $(2 \mathrm{~m} / \mathrm{s})$. (c) shows an example of an angle $\left(\mathbf{1 8 0}^{\circ}\right)$ where the SV mode and the SH mode waves are merged. Although a speed is identified on this image, visual assessment indicates that it is neither the SV nor the $\mathrm{SH}$ trajectory, and thus the speed estimate is excluded from the $\mathrm{SH}$ mode ellipse fitting.

the squared differences between the measured SH speeds and the predicted speeds from (11).

2D B-mode images from the transducer rotation angle $\phi_{\text {rot }}$ closest to fiber rotation $\phi_{\text {fiber }}$ were evaluated to determine the fiber tilt angle $\theta_{\text {tilt }}$. These B-mode images were analyzed using a two dimensional Fourier transform (2DFT) approach as seen in Fig. 5. We assumed that the fibers were represented by a series of parallel lines at a given tilt. We took the 2DFT of the $\mathrm{B}$-mode image and integrated radially over spatial frequencies 0.2 to $1 \mathrm{~mm}^{-1}$ within the 2DFT plane. The angle of peak signal was related to the fiber tilt in the B-mode using the rotation property of 2DFT as seen in Fig. 5(b).

Finally, estimates of $\mu_{L}, \mu_{T}$, and $\phi_{f i b e r}$, now accounting for $\theta_{\text {tilt }}$, were determined by repeating the fitting procedure using (10), but with the angle $\psi$ determined relative to the

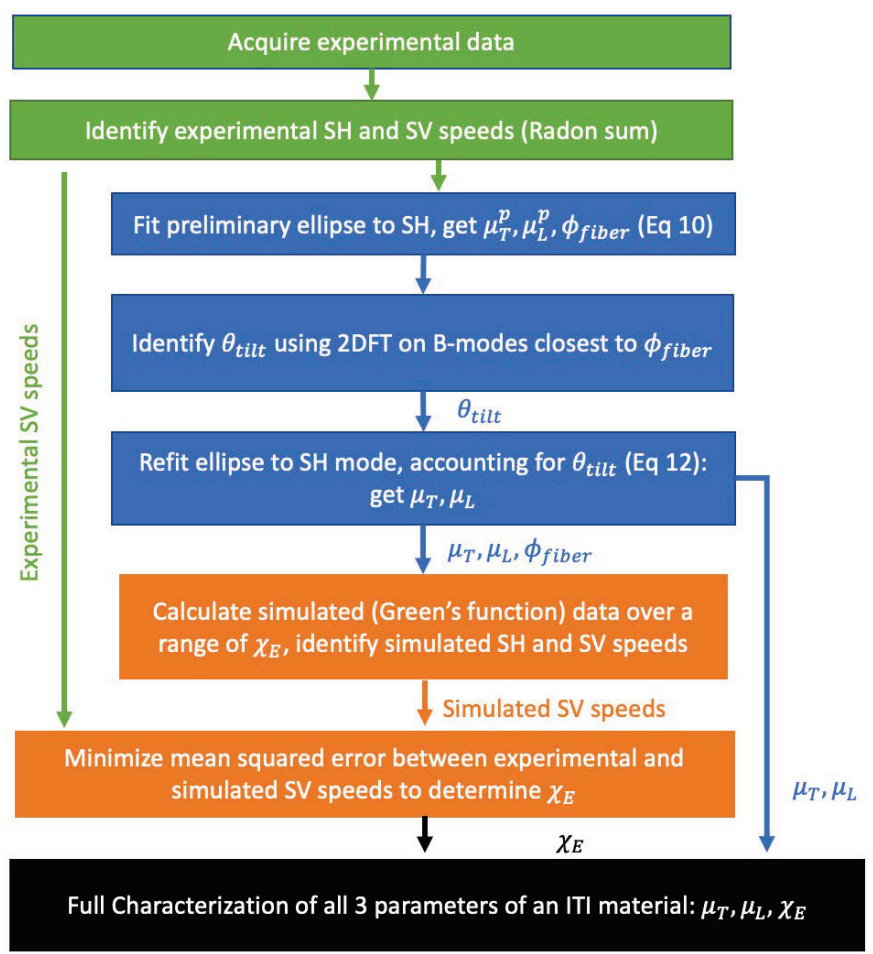

Fig. 4. Flowchart of data processing steps. The green boxes show acquiring and initial processing of the experimental data (Sections III-A and III-B). The blue steps show the steps for determining $\theta_{\text {tilt }}, \phi_{\text {fiber }}$, $\boldsymbol{\mu}_{L}$, and $\boldsymbol{\mu}_{T}$ (Section III-C). The orange boxes show the iterative parameter fitting steps that use the simulated Green's function (GF) data to determine $\chi_{E}$ (Section III-D.)

material symmetry axis,

$$
\psi=\cos ^{-1}(\widehat{A} \cdot \widehat{n})=\cos ^{-1}\left[\cos \left(\theta_{\text {tilt }}\right) \cos \left(\phi_{\text {rot }}-\phi_{\text {fiber }}\right)\right] \text {. }
$$

Additionally, estimates of shear anisotropy $\chi_{\mu}$ can be found at this point using (3) as $\mu_{L}$ and $\mu_{T}$ have been determined.

\section{Estimation of $\chi_{\mathrm{E}}$}

The tensile anisotropy $\chi_{E}$ was determined by simulating shear wave signals with Green's functions (GF) with matched $\mu_{L}$ and $\mu_{T}$ to the experimental data using a range of values for $\chi_{E}$ and comparing the SV mode shear wave speeds from these simulations to the measured in vivo SV mode speeds. The shear wave signals were calculated using Green's function techniques as described by Rouze et al. [30], using the ARFI excitation force calculated using Field II [57] modeling the experimental setup.

The final estimate of $\chi_{E}$ was obtained by calculating a series of $\mathrm{XY}$ plane simulated shear wave signals for a range of values of $\chi_{E}$ and selecting the value which minimized the mean squared error (MSE) between the simulated and experimentally measured SV speeds,

$$
M S E=\frac{1}{n} \sum_{i=1}^{n}\left(\left(V_{S V}^{E x p}\right)_{i}-\left(V_{S V}^{S i m}\right)_{i}\right)^{2}
$$

where $\mathrm{n}$ is the number of detected experimental SV speeds. In order to assess the accuracy of our ITI parameter estimation 

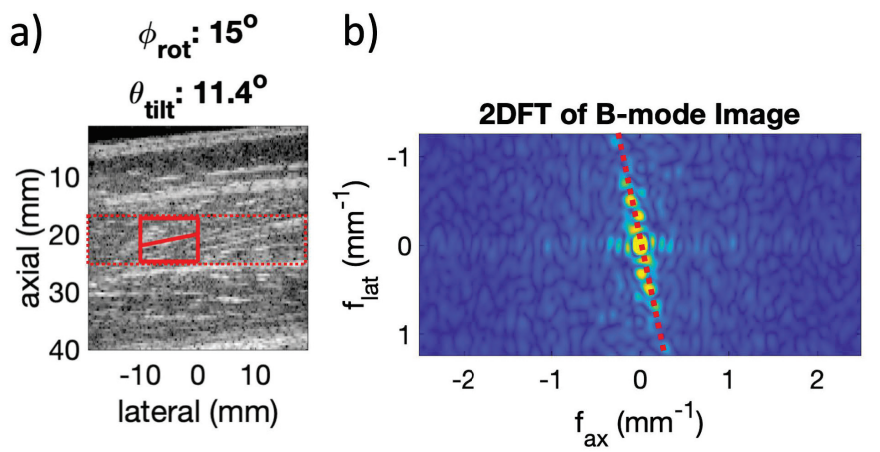

Fig. 5. a) shows an example B-mode for determining $\boldsymbol{\theta}_{\text {tilt }}$. The red box shows a sample ROI and resultant $\boldsymbol{\theta}_{\text {tilt }}$ estimate on the B-mode. The dotted red box shows the range of lateral positions included as the 10 $\mathrm{mm}$ box moved across the image to estimate standard deviation of tilt in the B-mode image. This tilt, $\boldsymbol{\theta}_{\text {tilt }}$, is then used in (12). b) shows the 2D FFT of the selected region from (a). The dotted red line shows the peak intensity representing the tilt of the fibers, $\boldsymbol{\theta}_{\text {tilt }}$, in this case $11.4^{\circ}$.

method, we used our previously validated FEM models to simulate shear wave propagation in an ITI material with known material parameters [29].

\section{Results}

Fig. 6 shows particle velocity shear wave signals from a single acquisition in an in vivo vastus lateralis muscle as a function of space and time for 18 transducer rotation angles spaced at $10^{\circ}$ rotation steps. As the transducer only rotates around $180^{\circ}$, at each rotation angle the left moving propagation direction and right moving propagation direction signals are separated, giving two signals per acquisition (36 signals shown in Fig. 6 obtained from 18 transducer rotation angles). This set represents one half of the signals acquired in a complete data set with $5^{\circ}$ rotation steps.

A key observation in Fig. 6 is that, as the transducer rotational position is varied, multiple shear wave signals are observed for rotational directions near $\phi_{\text {rot }}=-10^{\circ}, 30^{\circ}, 170^{\circ}$, and $210^{\circ}$, while only a single shear wave signal is observed for rotational angles near $\phi_{\text {rot }}=-90^{\circ}$ and $90^{\circ}$.

To see the difference between multiple trajectories better, enlarged space-time images of selected shear wave signals are shown in Fig. 3, corresponding to $\phi_{\text {rot }}=120^{\circ}, 40^{\circ}$, and $180^{\circ}$. Because the fiber tilt shown in Fig. 5(a) is small $\left(11.4^{\circ}\right)$, it is expected that the largest amplitude signal is the $\mathrm{SH}$ propagation mode. When a second, faster shear wave signal is seen, for example in Fig. 3(b), this mode is identified as the $\mathrm{SV}$ propagation mode.

In the center of Fig. 6 the shear wave speeds are plotted as a function of propagation angle with the $\mathrm{SH}$ propagation mode shown as orange triangles, and the SV propagation mode shown in red circles. For propagation directions near $\phi_{\text {rot }}=$ $15^{\circ}$ and $195^{\circ}$ the $\mathrm{SH}$ and $\mathrm{SV}$ trajectories on the space-time images appear to merge, and it was not possible to assign an estimated speed to either mode. These cases (blue squares) were omitted from further analysis.

From the B-mode image in Fig. 2(b) and the distributions of shear wave speeds for the SH propagation mode shown in the center of Fig. 6, it is clear that the material symmetry axis
$\widehat{A}$ in Fig. 1(b) is not aligned with the $\mathrm{X}$-axis $\left(0^{\circ} \rightarrow 180^{\circ}\right)$ for this dataset. Instead it is rotated by angle $\phi_{\text {fiber }}\left(16.5^{\circ}\right)$ about the $\mathrm{Z}$-axis and is also tilted by an angle of $\theta_{\text {tilt }}\left(11.4^{\circ}\right)$ relative to the $\mathrm{X}-\mathrm{Y}$ imaging plane.

For the data shown in Fig. 6, the nonlinear least squares fitting found the preliminary estimates of $c_{L}^{p}=2.25 \mathrm{~m} / \mathrm{s}$ $\left(\mu_{L}^{p}=5.07 \mathrm{kPa}\right)$, and $c_{T}^{p}=1.37 \mathrm{~m} / \mathrm{s}\left(\mu_{T}^{p^{L}}=1.89 \mathrm{kPa}\right)$. Note that these are not the speeds given in Fig. 6 as they are preliminary estimates. For this same acquisition, the 2DFT derived $\theta_{\text {tilt }}$ value from the $\phi_{\text {rot }}=15^{\circ}$ and $20^{\circ} \mathrm{B}$-mode data were weighted according to the proximity to $\phi_{\text {fiber }}$ with the result of $\theta_{\text {tilt }}=11.4^{\circ} \pm 2.5^{\circ}$. Once $\theta_{\text {tilt }}$ was accounted for geometrically, the final values of $c_{L}=2.33 \mathrm{~m} / \mathrm{s}, c_{T}=$ $1.37 \mathrm{~m} / \mathrm{s}, \mu_{L}=5.41 \mathrm{kPa}, \mu_{T}=1.89 \mathrm{kPa}, \phi_{\text {fiber }}=16.5^{\circ}$, and $\theta_{\text {tilt }}=11.4^{\circ}$ were found. These speeds are indicated on Fig. 6.

The top row of Fig. 7 shows simulated shear wave particle velocity signals calculated using Green's functions at a time of $3 \mathrm{~ms}$ post-push calculated using the values estimated by our algorithm $\left(\mu_{L}=5.41 \mathrm{kPa}, \mu_{T}=1.89 \mathrm{kPa}, \phi_{\text {fiber }}=\right.$ $16.5^{\circ}$, and $\theta_{\text {tilt }}=11^{\circ}$ ) for three values of tensile anisotropy, $\chi_{E}=3.31,5.18$, and 6.90. The middle row of Fig. 7 shows corresponding shear wave speeds. An important observation for the simulated signals is that the signal amplitude varies with propagation direction, particularly for the SV propagation mode. Even with the same underlying $\mu_{L}, \mu_{T}, \phi_{\text {fiber }}$, and $\theta_{\text {tilt }}$, small changes in $\chi_{E}$ affect the magnitude and speed of the SV wave.

The bottom row of Fig. 7 shows the shear wave speeds from experimental data (Fig. 6) overlaid on the calculated speeds from the simulated data (middle row). For these plots, the simulated SV speeds are color coded by the amplitude of the shear wave signal in the top row. We observe that the speeds determined from the signals with the largest shear wave amplitude occur at transducer angles near $\phi_{\text {rot }}=-10^{\circ}, 40^{\circ}, 170^{\circ}$, and $220^{\circ}$, which are roughly the same directions where two shear wave signals are observed experimentally. The iterative parameter estimation technique using Green's Functions gave $\chi_{E}=5.20$ for the acquisition shown in Fig. 6. The fact that most of the large amplitude SV signals in the simulated data are concordant with the experimental data adds confidence to the biofidelity of this tensile anisotropy $\chi_{E}$ estimation approach.

In four acquisitions on two different days in the same location on the vastus lateralis of a healthy volunteer, four estimates of $\mu_{L}, \mu_{T}, \chi_{\mu}$, and $\chi_{E}$ were calculated as shown in Fig. 8. The mean and standard deviation values for $\mu_{L}, \mu_{T}$, $\chi_{\mu}, \chi_{E}$, and MSE between Green's function simulations and experimental data from (13) are given in Table II.

\begin{tabular}{|c|c|}
\hline$\mu_{L}$ & $5.77 \pm 1.00 k P a$ \\
$\mu_{T}$ & $1.93 \pm 0.41 k P a$ \\
$\chi_{\mu}$ & $2.11 \pm 0.92$ \\
$\chi_{E}$ & $4.67 \pm 1.40$ \\
$M S E$ & $0.34 \pm 0.20(\mathrm{~m} / \mathrm{s})^{2}$ \\
\hline
\end{tabular}

TABLE ॥

MEAN AND STANDARD DEVIATION ACROSS 4 ACQUISITIONS in VIVO IN THE vastus lateralis ACROSS 2 DAYS IN 1 HEALTHY VOLUNTEER 


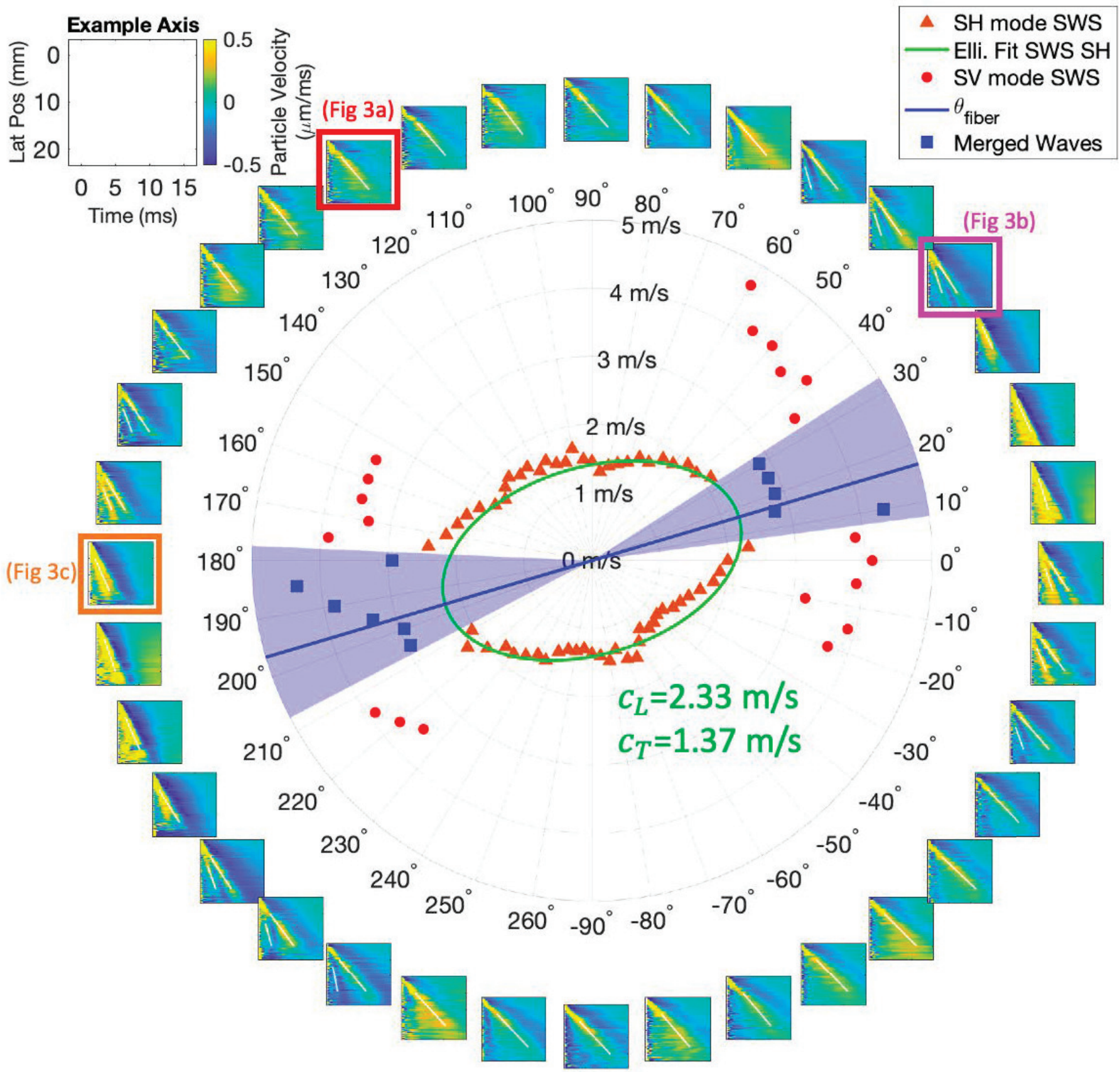

Fig. 6. Space time plots and speeds at many angles. The subplots going around the ring show space-time plots in $10^{\circ}$ increments of $\phi_{\text {rot }}$ (transducer rotation). An example axis label for each subplot is shown in the upper left. The white lines on each subplot identify the trajectory of speeds identified. Each space time plot contains 0,1 , or 2 trajectories. The number of waves identified and calculated speeds are seen in the inner circle plot. The slower SH mode wave speeds are seen in orange triangles, while the faster SV speeds are in red circles. The ellipse fit to the SH speeds is shown in green (using (11) and (12)), as is the speeds along the major axis of the ellipse $\left(c_{L}\right)$, and the minor axis of the ellipse $\left(c_{T}\right)$. The blue line shows the rotational angle of the fibers $\phi_{\text {fiber }}$ corresponding to the major axis of the ellipse. These values of $c_{L}$ and $c_{T}$ are after $\boldsymbol{\theta}_{\text {tilt }}$ has been accounted for (see Section III-C.). The blue squares and blue shading indicate speeds that were excluded by our processing as they appeared as merged waves (see Fig. 3(c)), but are displayed to show what would have been measured by a 2D SWEI system.

As seen in Fig. 9, the predicted $\chi_{E}$ increases monotonically with tilt. A tilt accuracy of $\pm 2.50^{\circ}$ was determined by sliding the ROI across the B-mode image laterally and assessing fiber angles. We then computed GF simulations for the range of $\theta_{\text {tilt }}$ values measured and found a precision of $\pm 7.4 \%$ for $\chi_{E}$ estimates.

Fig. 10 shows good concordance between the matched FEM and GF simulations. Similar SV wave speeds were found, particularly at angles $\phi_{\text {fiber }}$ where the SV wave was strongest, which is also where the experimental SV speeds were measured. There was $0.11 \pm 0.13 \mathrm{~m} / \mathrm{s}$ difference between GF and FEM for SV wave speeds, and $0.07 \pm 0.07 \mathrm{~m} / \mathrm{s}$ difference between GF and FEM for SH wave speeds. When the speeds of the SV waves found in FEM simulation were used in the reconstruction algorithm described in Fig. 4 and Section III-D, the resultant $\chi_{E}$ for the FEM data was found to 

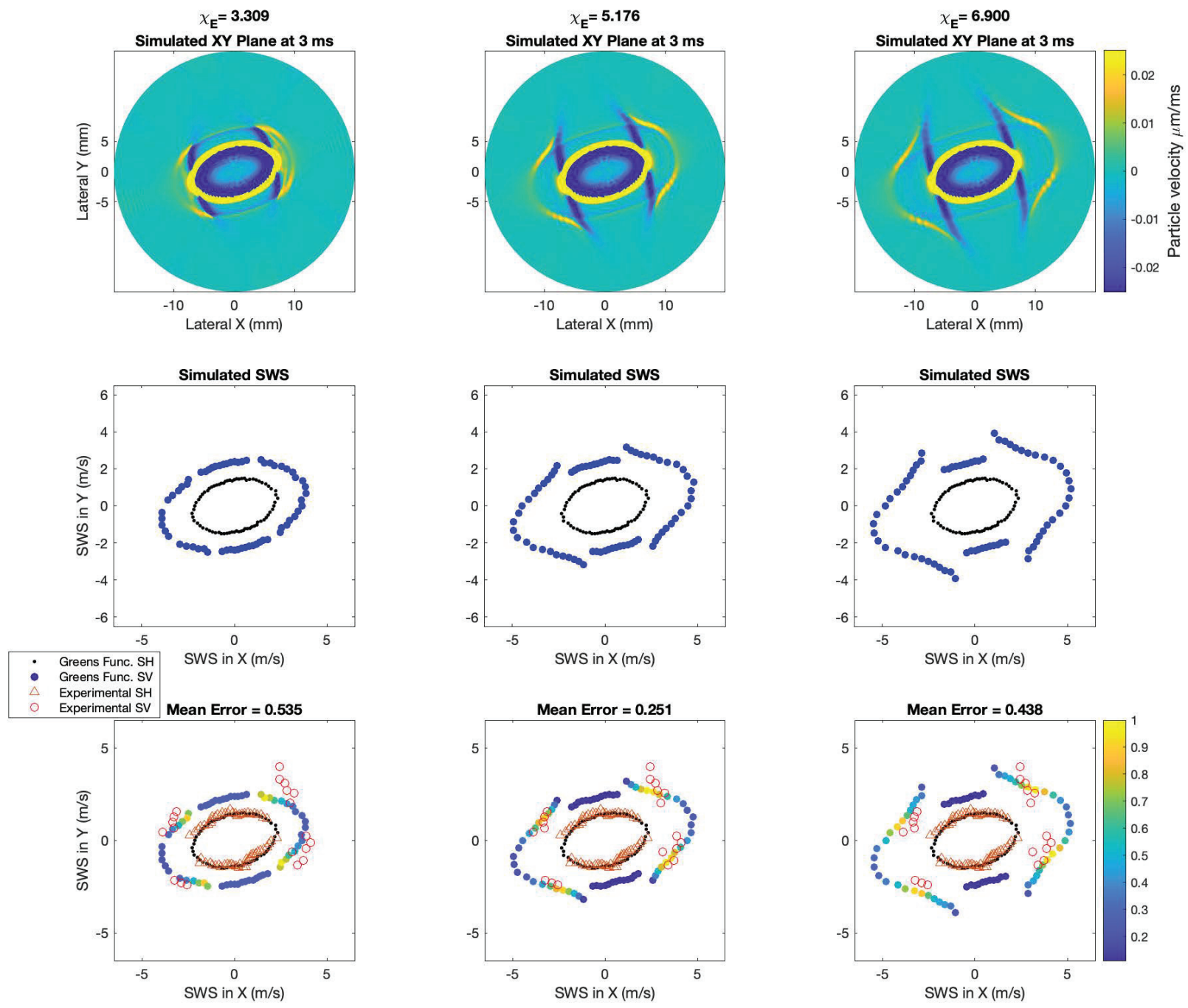

Fig. 7. Comparison of simulated and experimental signals. The top row shows simulated Green's function signals in the $\mathrm{XY}$ plane at at time of 3 $\mathrm{ms}$ from three different values of $\chi_{E}$ for fixed values of $\mu_{L}, \mu_{T}, \phi_{\text {fiber }}$, and $\boldsymbol{\theta}_{\text {tilt }}$. In the middle row and bottom row, the simulated SH (black dots) and SV speeds (blue dots) at each angle are shown. In the bottom row the experimental SH mode is shown in the open orange triangles, and the experimental SV mode is shown in open red circles. In the bottom row the colors of the solid circles show the SV mode strength relative to the other SV mode speeds present in the simulations (color bar max at 1), as calculated by normalizing each by the maximum Radon sum value found across all SV mode speeds. Yellow indicates a strong SV mode relative to blue. The fact that most of the high amplitude signals (yellow dots) are concordant with the experimental data adds confidence to the biofidelity of this $\chi_{E}$ estimation approach.

be 5.30, while the input $\chi_{E}$ to the FEM simulation was 5.25, and the $\chi_{E}$ found from the matching experimental data from which the input $\mu_{L}$ and $\mu_{T}$ FEM parameters were drawn was $\chi_{E}=5.2$, representing a $2 \%$ difference in $\chi_{E}$ from FEM to experimental.

\section{DISCUSSION}

In this paper, we demonstrate that measuring both the SV and $\mathrm{SH}$ wave modes is possible using 3D SWEI in in vivo muscle by leveraging the tilted geometry of the muscle fibers relative to the transducer face. We also present a method of estimating the tensile anisotropy $\chi_{E}$ of muscle.

Fig. 7, bottom middle panel, demonstrates the agreement between the GF predicted $\mathrm{SV}$ and $\mathrm{SH}$ speeds and the in vivo experimental measurements. In the middle column, not only is the error between the GF and the experimental speeds minimized, but the location of the largest amplitude simulated SV signals (yellow and green dots) align with the angles where the experimental SV speeds were observed. The appearance of SV waves experimentally at the four regions where the GF predict the largest amplitude SV waves increases confidence that SV waves are not reflected waves.

As seen in Fig. 8, in each of four acquisitions on two days in the same healthy volunteer, the $\mu_{L}$ value measured was greater than $\mu_{T}$. Additionally, the tensile anisotropy $\chi_{E}$ was greater than the shear anisotropy $\chi_{\mu}$ in each acquisition.

As seen in Fig. 9, as the tilt angle $\theta_{\text {tilt }}$ increases, the 

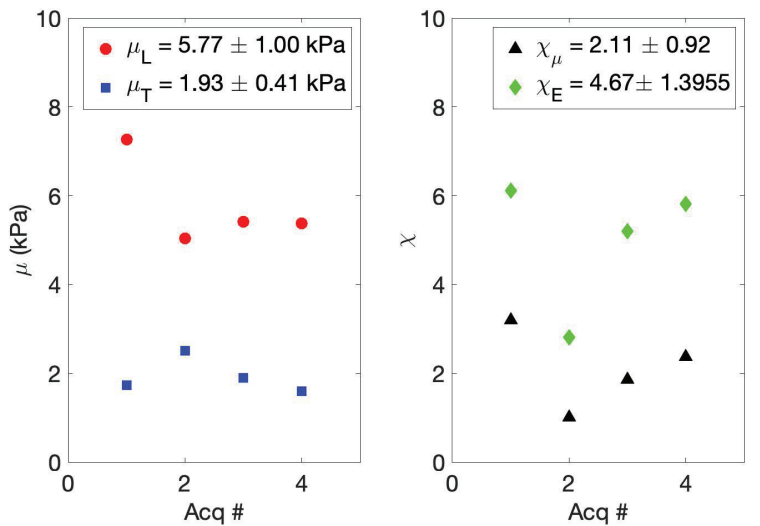

Fig. 8. Reconstructed ITI material properties from four separate datasets acquired on two different days in the vastus lateralis of a healthy volunteer. In each acquisition $\mu_{L}>\mu_{T}$ and $\chi_{E}>\chi_{\mu}$.

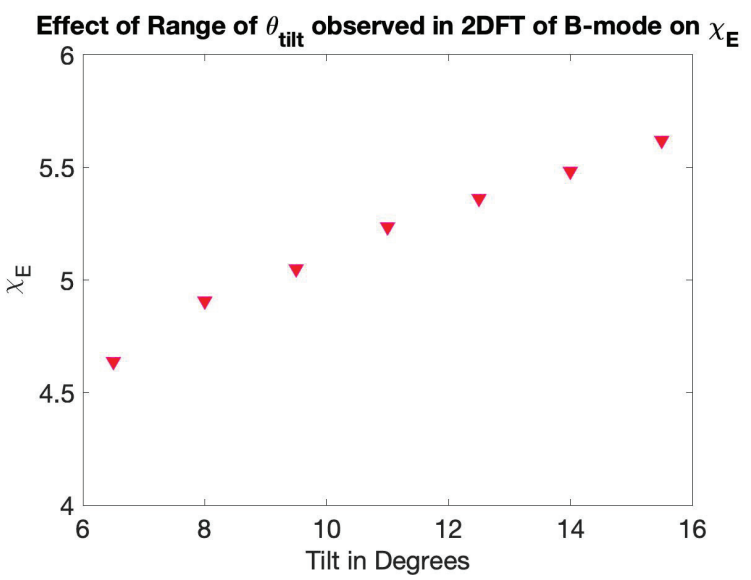

Fig. 9. Effect of variation in $\boldsymbol{\theta}_{\text {tilt }}$ on $\chi_{E}$.

calculated $\chi_{E}$ value also increases monotonically, indicating expected bias if the tilt angle is not correctly computed. However, across a $95 \%$ confidence interval of the $\theta_{\text {tilt }}=$ $11.4 \pm 2.5^{\circ}$ measured in the B-mode data, the variation of $\chi_{E}(0.344)$ represents only a $6 \%$ bias.

To quantify the expected accuracy of our Green's function approach to estimating $\chi_{E}$, for one acquisition, FEM simulations were computed to match the experimental setup with known inputs. As seen in Fig. 10, the matched GF and FEM simulated speeds at angles where there were experimentally observed SV waves varied from each other by only $0.11 \pm 0.13$ $\mathrm{m} / \mathrm{s}$, while the SH wave speeds differences between the GF and FEM were $0.07 \pm 0.07 \mathrm{~m} / \mathrm{s}$. Additionally, using the Green's function reconstruction algorithm for this data set found an estimate of $\chi_{E}=5.30$, while the ground truth value was $\chi_{E}=$ 5.25 , meaning a difference of $1 \%$. Using a computational cluster, the GF plane calculations of $\mathrm{Z}$ direction displacement and resultant SWS in the XY plane necessary to estimate 120 possible $\chi_{E}$ values for a given $\theta_{t i l t}, \mu_{L}$, and $\mu_{T}$ took approximately $150 \mathrm{CPU}$-hours on an Intel@Xeon Gold $3 \mathrm{GHz}$ $\mathrm{CPU}$. We are exploring methods to pre-populate look up tables to reduce this time, but this work is outside the scope of this paper.

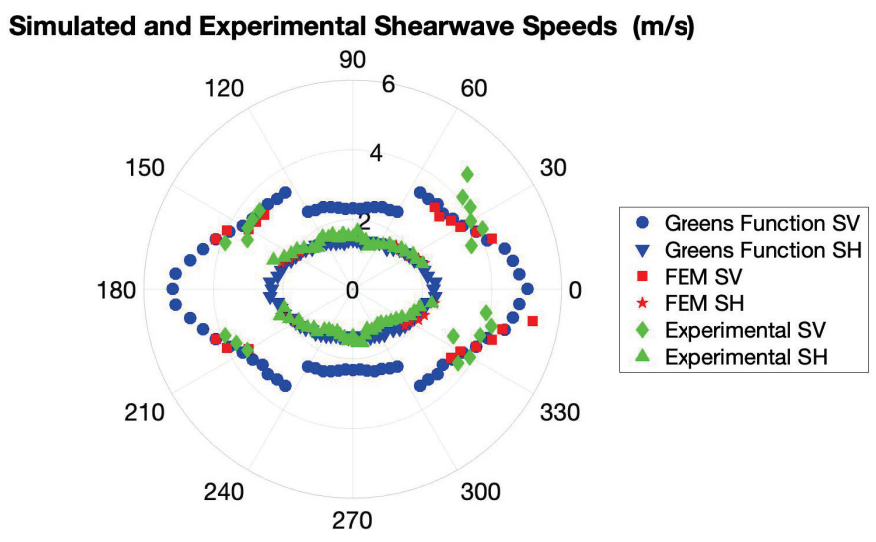

Fig. 10. Validation with Finite Element Method (FEM). For one example acquisition, FEM simulations to match at angles where experimental SV waves were measured.

The relationship between shear modulus and Young's modulus in an isotropic, elastic, incompressible material is $E=3 \mu$. However in ITI materials, there is no simple proportional relationship between $\mathrm{E}$ and $\mu$ in any given direction. As seen in Fig. 8, and as predicted by the equations modeling ITI materials (2)-(3), (5)-(7), $\chi_{E}$ is not equal to $\chi_{\mu}$ and thus represents an additional independent parameter that may be explored for muscle health characterization.

One of the advantages of a rotational 3D approach is that since all rotational angles are captured in $5^{\circ}$ increments, the 3D SWEI methodology presented herein is completely agnostic to the starting position of the fiber rotation $\phi_{\text {fiber }}$ relative to the transducer. This means the 3D SWEI approach can be used without first identifying muscle fiber orientation with freehand B-mode imaging.

The values for the shear modulus along the fibers $\left(\mu_{L}=5.77 \pm 1.00 \mathrm{kPa}\right)$ are similar but slightly higher than those found by other studies in the vastus lateralis using 2D SWEI $(4.5 \pm 1.0 \mathrm{kPa}$ [28], $3.6 \pm 0.45 \mathrm{kPa}$ [7], $3.3 \pm 0.4 \mathrm{kPa}$ [58], and $5.1 \pm 1.3 \mathrm{kPa}$ [59]). The use of 3D data herein ensures the $\mu_{L}$ and $\mu_{T}$ measurements account for both the rotation $\phi_{\text {fiber }}$ and tilt $\theta_{\text {tilt }}$ angles of the muscle fibers. Misalignment in either dimension would result in an underestimation of $\mu_{L}$, which could be a source of bias in the results of $2 \mathrm{D}$ SWEI studies. In particular, accounting for the tilt angle $\theta_{\text {tilt }}$ in (12) increases our estimates. On average $\mu_{L}$ increased by $7.6 \% \pm 3.5 \%$ when $\theta_{\text {tilt }}$ was incorporated, as compared to the assumption $\theta_{\text {tilt }}=0^{\circ}$.

As seen in the center of Fig. 6, the speeds closest to the major axis of the SH mode ellipse were not included in the ellipse fitting. At those locations, visual assessment of the space-time plots made it clear that the SV and SH modes were spatially overlapping with each other. While the Radon sum algorithm was able to identify a trajectory, visual assessment could not determine if the trajectory was calculated using only SV mode data, only SH mode data, or a combination of both SV and SH mode, so the speed was excluded from further analysis. Supporting this, as seen in Fig. 7 bottom row, directly along the major axis of the ellipse, the SV speed predicted by simulation is very low amplitude compared to other SV speeds at other angles. When the inherent noise of 
experimental motion tracking is combined with this effect, it is unsurprising that it is difficult to separate the SH and SV modes for these propagation directions in experimental data.

The difficulty in separating the SH and SV waves along the main fiber axis could also be one of many factors contributing to the wide variability in speeds found using 2D SWEI techniques [24]. If the method used to identify the shear wave speed along the fibers does not account for the presence of both SV and SH mode speeds, it is unknown whether the speed measured along the fiber axis is from the $\mathrm{SH}$ mode or the SV mode, increasing variability in the measurements. The blue squares and highlighted area of Fig. 6 indicate what speed would be estimated by 2D SWEI if the delineation of SH and SV waves was not considered. Our work overcomes this by informing the estimate of $\mu_{L}$ with speeds around the ellipse rather than relying solely on speeds aligned with the fiber dimension, and inspecting space-time plots for multiple wave fronts.

As a proof of concept work, this paper presents measurements from only one healthy volunteer. However, the repeatability across multiple days and multiple acquisitions supports the feasibility of this method and motivates the development of 3D SWEI for muscle imaging and measuring mechanical properties of muscle.

The concordance between the Green's function simulations and in vivo muscle data demonstrate that the linear, elastic, ITI model is a reasonable model in the vastus lateralis of a healthy volunteer. The ability to extend this model to other muscles is limited by the size of the muscle, as when the thickness of the muscle body approaches the shear wavelength it is likely that other wave guiding behavior and boundary effects would influence waves measured. Additionally, a limitation of this method is the requirement that the experimental and simulated parameters are exactly matched, as any mismatches would be expected to introduce bias in the $\chi_{E}$ estimates.

A notable limitation of this work is that it neglects viscosity. Viscosity introduces dispersion and attenuation in shear wave elastography data, which has been shown to bias estimated parameters based upon group speeds [60]. We have observed some dispersion in vastus lateralis data, primarily across the fibers with less dispersion along the fibers [61], which is consistent with reports from the literature [45]. Thus, it is possible that the SV mode analysis presented here has some bias, which might be addressed at the expense of computational complexity, but is outside the scope of this initial demonstration.

For years, three parameter models of ITI materials have been proposed for muscle SWEI modeling [29], [30], [46], but to date quantification of all three parameters has not been realized in in vivo ultrasound SWEI. Recent demonstration of the ability to resolve two wave fronts in phantoms [51] showed the feasibility of using a tilted excitation to reconstruct tensile anisotropy.

Additionally, Li et al. have investigated these phenomena in biceps brachii and gastrocnemius muscles using 2D SWEI and measurements at 3 specified orientations of the transducer, assuming that the group shear wave speed measured with the transducer at $\theta_{\text {tilt }}=45^{\circ}$ represents the SV mode [62].
They found values that result in a $\chi_{\mu}$ of 1.53 in the biceps brachii and 0.31 in the gastrocnemius, and $\chi_{E}$ of 0.75 in the biceps brachii and 0.81 in the gastrocnemius. All of these values are about 4 times lower than the range of values reported for the vastus lateralis herein. However they also measured a mean $v_{S V}$ of $1.8 \mathrm{~m} / \mathrm{s}$, while we found a mean $v_{S V}$ of $3.8 \mathrm{~m} / \mathrm{s}$, and given the relationship between $v_{S V}^{2}$ and $\chi_{E}$ seen in (9), it is unsurprising that approximately doubling the SV speed leads to a quadrupling of $\chi_{E}$, showing again the importance of accurately measuring the SV speed. The 3D SWEI method herein leverages the tilt concepts but also facilitates characterization in muscles with a range of interrogation angles and fiber orientations.

Three ITI material parameters have been measured in vivo by magnetic resonance elastography (MRE) [48], and in ex vivo animal tissue [49], [50]. Looking at the three muscles in the calf using MRE in vivo, Guo et al. [48] found lower $\chi_{\mu}$ values (soleus 0.50 , gastrocnemius 0.80 , tibialis anterior 0.27 ) compared to $\chi_{\mu}=2.11$ herein for vastus lateralis, and also $\chi_{E}$ values lower than herein (soleus 1.47 , gastrocnemius 2.26 , tibialis 2.00, compared to $\chi_{E}=4.67$ for vastus lateralis). These lower values for both tensile and shear anisotropy indicate the muscles appeared more isotropic using MRE than with 3D SWEI. It is encouraging that $\chi_{E}>\chi_{\mu}$ with both MRE and 3D SWEI. There are several factors that could account for differences between these results. The interrogated muscles reported are different. The nature of the external continuous wave vibration and inversion of the wave equation employed by MRE means the parameters reconstructed are not as localized within the muscle, whereas 3D rotational SWEI measurements of muscle apply to a smaller region of interest $\left(4 \mathrm{~cm}^{2}\right)$. Additionally, the frequency content of MRE in the cited studies $(40 \mathrm{~Hz}, 50 \mathrm{~Hz}$, and $60 \mathrm{~Hz}$ in Guo et al.) is lower than the frequency content of SWEI used here (100-400 $\mathrm{Hz}$ ). As frequency increases shear dissipative effects increase. Thus, there is an expectation that moduli estimated based on an elastic assumption will have an increasing bias as frequency increases. For example, see Yasar et al. [63], which shows that typically both the shear storage and loss moduli monotonically increase with excitation frequency.

The ability to measure tensile anisotropy $\chi_{E}$ and $\chi_{\mu}$ may provide a unique biomarker for muscle characterization. However, the relationship between these material parameters, as well as $\mu_{L}$ and $\mu_{T}$, and muscle health remains an open question. While still only a proof of concept, determining these relationships has potential application in characterizing a wide variety of muscle diseases including myopathies, dystrophies, muscle spasticities, muscle atrophy, age related sarcopenia, and athletic injury repair and healing.

\section{CONCLUSION}

In this study, we describe measurements of multiple shear wave propagation modes in in vivo muscle using a 3D SWEI experimental setup with a tilted material symmetry axis, shown in Fig. 1(b). Results are reported from measurements made in a healthy volunteer on two different days in the relaxed vastus lateralis muscle, where the fibers are naturally tilted 
with respect to the skin surface. We demonstrate 3D SWEI can improve estimation of $\mu_{L}$ and $\mu_{T}$ by accounting for the effects of fiber rotation $\phi_{\text {fiber }}$ and tilt $\theta_{\text {tilt }}$ when evaluating SH mode speeds, which is not possible in 2D SWEI. We also demonstrate 3D SWEI measurement of the SV propagation mode which, when combined with matched Green's function simulations, allows for estimation of tensile anisotropy, $\chi_{E}$.

\section{REFERENCES}

[1] A. Sarvazyan, T. J. Hall, M. W. Urban, M. Fatemi, S. R. Aglyamov, and B. S. Garra, "an Overview of Elastography - an Emerging Branch of Medical Imaging," Current medical imaging reviews, vol. 7, no. 4, pp. 255-282, 2011.

[2] J. R. Doherty, G. E. Trahey, K. R. Nightingale, and M. L. Palmeri, "Acoustic Radiation Force Elasticity Imaging in Diagnostic Ultrasound," IEEE Transactions on Ultrasonics, Ferroelectrics and Frequency Control, vol. 60, no. 4, 2013.

[3] R. M. Sigrist, J. Liau, A. E. Kaffas, M. C. Chammas, and J. K. Willmann, "Ultrasound elastography: Review of techniques and clinical applications," Theranostics, vol. 7, no. 5, pp. 1303-1329, 2017.

[4] A. P. Sarvazyan et al., "Shear Wave Elasticity Imaging: A New U1trasonic Technology of Medical Diagnostics," Ultrasound Med. Biol., vol. 24, no. 9, pp. 1419-1435, 1998

[5] M. L. Palmeri and K. R. Nightingale, "Acoustic radiation force-based elasticity imaging methods," Interface Focus, vol. 1, pp. 553-564, 2011.

[6] T. Shiina et al., "WFUMB guidelines and recommendations for clinical use of ultrasound elastography: Part 1: Basic principles and terminology," Ultrasound in Medicine and Biology, vol. 41, no. 5, pp. 11261147, 2015.

[7] C. Zhang and others., "Diagnostic Value of Virtual Touch Tissue Imaging Quantification for Evaluating Median Nerve Stiffness in Carpal Tunnel Syndrome," Journal of Ultrasound in Medicine, pp. 1783-1791, 2017.

[8] S. F. Eby et al., "Shear wave elastography of passive skeletal muscle stiffness: Influences of sex and age throughout adulthood," Clinical Biomechanics, vol. 30, no. 1, pp. 22-27, 2015.

[9] S. Mackintosh, A. Young, A. Lee, and J. Sim, "Considerations in the application of two dimensional shear wave elastography in muscle," no. March, pp. 1-9, 2019.

[10] A. M. Alfuraih, A. L. Tan, P. O'Connor, P. Emery, and R. J. Wakefield, "The effect of ageing on shear wave elastography muscle stiffness in adults," Aging Clinical and Experimental Research, vol. 31, no. 12, pp. $1755-1763,2019$.

[11] K. M. M. e. Lima, J. F. S. Costa Júnior, W. C. d. A. Pereira, and L. F. De Oliveira, "Assessment of the mechanical properties of the muscletendon unit by supersonic shear wave imaging elastography: A review," Ultrasonography, vol. 37, no. 1, pp. 3-15, 2018.

[12] Y. Yoshitake, Y. Takai, H. Kanehisa, and M. Shinohara, "Muscle shear modulus measured with ultrasound shear-wave elastography across a wide range of contraction intensity," Muscle and Nerve, vol. 50, no. 1, pp. 103-113, 2014.

[13] M. Shinohara, K. Sabra, J. L. Gennisson, M. Fink, and M. L. Tanter, "Real-time visualization of muscle stiffness distribution with ultrasound shear wave imaging during muscle contraction," Muscle and Nerve, vol. 42, no. 3, pp. 438-441, 2010.

[14] L. A. Chernak, R. J. Dewall, K. S. Lee, and D. G. Thelen, "Length and activation dependent variations in muscle shear wave speed," Physiological Measurement, vol. 34, no. 6, pp. 713-721, 2013.

[15] A. M. Alfuraih et al., "Muscle shear wave elastography in idiopathic inflammatory myopathies: a case-control study with MRI correlation," Skeletal Radiology, vol. 48, no. 8, pp. 1209-1219, 2019.

[16] E. L. Carpenter, H. A. Lau, E. H. Kolodny, and R. S. Adler, "Skeletal Muscle in Healthy Subjects versus Those with GNE-Related Myopathy: Evaluation with Shear-Wave US-A Pilot Study.” Radiology, vol. 277, no. 2, pp. 546-554, 112015 .

[17] A. Pichiecchio et al., "Muscle ultrasound elastography and MRI in preschool children with Duchenne muscular dystrophy," Neuromuscular Disorders, vol. 28, no. 6, pp. 476-483, 2018.

[18] C. J. Moore et al., "In Vivo Viscoelastic Response (VisR) Ultrasound for Characterizing Mechanical Anisotropy in Lower-Limb Skeletal Muscles of Boys with and without Duchenne Muscular Dystrophy," Ultrasound in Medicine and Biology, vol. 44, no. 12, pp. 2519-2530, 2018.

[19] S. F. Eby et al., "Quantifying spasticity in individual muscles using shear wave elastography," Radiology Case Reports, vol. 12, no. 2, pp. 348-352, 2017.
[20] J. E. Brandenburg et al., "Quantifying passive muscle stiffness in children with and without cerebral palsy using ultrasound shear wave elastography." Developmental medicine and child neurology, vol. 58, no. 12, pp. 1288-1294, 122016.

[21] J. E. Brandenburg, S. F. Eby, P. Song, W. R. Bamlet, G. C. Sieck, and K.N. An, "Quantifying Effect of Onabotulinum Toxin A on Passive Muscle Stiffness in Children with Cerebral Palsy Using Ultrasound Shear Wave Elastography." American journal of physical medicine \& rehabilitation, vol. 97 , no. 7, pp. 500-506, 72018.

[22] L. Mathevon et al., "Two-dimensional and shear wave elastography ultrasound: A reliable method to analyse spastic muscles?" Muscle and Nerve, vol. 57, no. 2, pp. 222-228, 2018.

[23] B. C. W. Kot, Z. J. Zhang, A. W. C. Lee, V. Y. F. Leung, and S. N. Fu, "Elastic Modulus of Muscle and Tendon with Shear Wave Ultrasound Elastography: Variations with Different Technical Settings," PLoS ONE, vol. 7, no. 8, 2012

[24] M. Creze, A. Nordez, M. Soubeyrand, L. Rocher, X. Maître, and M. F. Bellin, "Shear wave sonoelastography of skeletal muscle: basic principles, biomechanical concepts, clinical applications, and future perspectives," Skeletal Radiology, vol. 47, no. 4, pp. 457-471, 2018.

[25] K. Chino and H. Takahashi, "Influence of pennation angle on measurement of shear wave elastography: In vivo observation of shear wave propagation in human pennate muscle," Physiological Measurement, vol. 39 , no. 11,2018

[26] C. Dorado Cortez, L. Hermitte, A. Ramain, C. Mesmann, T. Lefort, and J. B. Pialat, "Ultrasound shear wave velocity in skeletal muscle: A reproducibility study," Diagnostic and Interventional Imaging, vol. 97, no. 1, pp. 71-79, 2016.

[27] A. M. Alfuraih, P. O'Connor, E. Hensor, A. L. Tan, P. Emery, and R. J. Wakefield, "The effect of unit, depth, and probe load on the reliability of muscle shear wave elastography: Variables affecting reliability of SWE," Journal of Clinical Ultrasound, vol. 46, no. 2, pp. 108-115, 2018.

[28] G. Dubois et al., "Reliable Protocol for Shear Wave Elastography of Lower Limb Muscles at Rest and During Passive Stretching," Ultrasound in Medicine and Biology, vol. 41, no. 9, pp. 2284-2291, 2015.

[29] N. C. Rouze, M. H. Wang, M. L. Palmeri, and K. R. Nightingale, "Finite element modeling of impulsive excitation and shear wave propagation in an incompressible, transversely isotropic medium." Journal of biomechanics, pp. 1-8, 92013.

[30] N. C. Rouze, M. L. Palmeri, and K. R. Nightingale, "Tractable calculation of the Greens tensor for shear wave propagation in an incompressible, transversely isotropic material," Phys Med Biol, vol. 65, pp. $1-17,2020$.

[31] W. M. Lai, D. Rubin, and K. Erhard, Introduction to Continum Mechanics, fourth edi ed. Elsevier Ltd., 2010.

[32] M. J. P. Musgrave, "The propagation of elastic waves in crystals and other anisotropic media," vol. 74, pp. 229-241, 1959

[33] J. L. Rose, Ultrasonic Waves in Solid Media, 1999.

[34] S. Chatelin, M. Bernal, C. Papadacci, J. L. Gennisson, M. Tanter, and M. Pernot, "Anisotropic polyvinyl alcohol hydrogel phantom for shear wave elastography in fibrous biological soft tissue," IEEE International Ultrasonics Symposium, IUS, pp. 1857-1860, 2014.

[35] D. J. Tweten, R. J. Okamoto, J. L. Schmidt, J. R. Garbow, and P. V. Bayly, "Estimation of material parameters from slow and fast shear waves in an incompressible, transversely isotropic material," Journal of Biomechanics, vol. 48, no. 15, pp. 4002-4009, 2015. [Online]. Available: http://www.sciencedirect.com/science/article/pii/S0021929015005035

[36] S. Aristizabal et al., "Shear wave vibrometry evaluation in transverse isotropic tissue mimicking phantoms and skeletal muscle." Physics in medicine and biology, vol. 59, no. 24, pp. 7735-7752, 122014.

[37] S. F. Eby, P. Song, S. Chen, Q. Chen, J. F. Greenleaf, and K. N. An, "Validation of shear wave elastography in skeletal muscle," Journal of Biomechanics, vol. 46, no. 14, pp. 2381-2387, 2013.

[38] S. Eby et al., "Quantitative Evaluation of Passive Muscle Stiffness in Chronic Stroke." American journal of physical medicine \& rehabilitation, vol. 95, no. 12, pp. 899-910, 122016.

[39] L. Ruby et al., "Which Confounders Have the Largest Impact in Shear Wave Elastography of Muscle and How Can They be Minimized? An Elasticity Phantom, Ex Vivo Porcine Muscle and Volunteer Study Using a Commercially Available System," Ultrasound in Medicine and Biology, vol. 45, no. 10, pp. 2591-2611, 2019.

[40] M. S. Taljanovic et al., "Shear-wave elastography: Basic physics and musculoskeletal applications," Radiographics, vol. 37, no. 3, pp. 855870, 2017.

[41] J. E. Brandenburg et al., "Ultrasound elastography: the new frontier in direct measurement of muscle stiffness." Archives of physical medicine and rehabilitation, vol. 95, no. 11, pp. 2207-2219, 112014. 
[42] E. E. Drakonaki, G. M. Allen, and D. J. Wilson, "Ultrasound elastography for musculoskeletal applications," British Journal of Radiology, vol. 85, no. 1019, pp. 1435-1445, 2012.

[43] M. Goo, L. M. Johnston, F. Hug, and K. Tucker, "Systematic Review of Instrumented Measures of Skeletal Muscle Mechanical Properties: Evidence for the Application of Shear Wave Elastography with Children," Ultrasound in Medicine \& Biology, vol. 00, no. 00, pp. 1-10, 2020.

[44] J.-L. Gennisson, S. Catheline, S. Chaffai, and M. Fink, "Transient elastography in anisotropic medium: Application to the measurement of slow and fast shear wave speeds in muscles," The Journal of the Acoustical Society of America, vol. 114, no. 1, pp. 536-541, 2003.

[45] J.-L. Gennisson, T. Deffieux, E. Macé, G. Montaldo, M. Fink, and M. Tanter, "Viscoelastic and anisotropic mechanical properties of in vivo muscle tissue assessed by supersonic shear imaging." Ultrasound in medicine \& biology, vol. 36, no. 5, pp. 789-801, 52010.

[46] D. Royer, J.-L. Gennisson, T. Deffieux, and M. Tanter, "On the elasticity of transverse isotropic soft tissues (L)," The Journal of the Acoustical Society of America, vol. 129, no. 5, pp. 2757-2760, 2011.

[47] S. Papazoglou, J. Rump, J. Braun, and I. Sack, "Shear wave group velocity inversion in MR elastography of human skeletal muscle," Magnetic Resonance in Medicine, vol. 56, no. 3, pp. 489-497, 2006.

[48] J. Guo, S. Hirsch, M. Scheel, J. Braun, and I. Sack, "Three-parameter shear wave inversion in MR elastography of incompressible transverse isotropic media: Application to in vivo lower leg muscles," Magnetic Resonance in Medicine, vol. 75, no. 4, pp. 1537-1545, 2016.

[49] J. L. Schmidt et al., "Magnetic resonance elastography of slow and fast shear waves illuminates differences in shear and tensile moduli in anisotropic tissue." Journal of biomechanics, vol. 49, no. 7, pp. 10421049, 52016.

[50] C. A. Guertler et al., "Estimation of Anisotropic Material Properties of Soft Tissue by MRI of Ultrasound-Induced Shear Waves," Journal of Biomechanical Engineering, vol. 142, no. 3, pp. 1-17, 2020.

[51] A. Caenen, A. E. Knight, N. C. Rouze, N. B. Bottenus, P. Segers, and K. R. Nightingale, "Analysis of multiple shear wave modes in a nonlinear soft solid : Experiments and finite element simulations with a tilted acoustic radiation force," Journal of the Mechanical Behavior of Biomedical Materials, vol. 107, no. January, p. 103754, 2020.

[52] M. Wang, B. Byram, M. Palmeri, N. Rouze, and K. Nightingale, "Imaging transverse isotropic properties of muscle by monitoring acoustic radiation force induced shear waves using a 2-D matrix ultrasound array," IEEE Transactions on Medical Imaging, vol. 32, no. 9, pp. 16711684, 2013.

[53] Y. Deng, N. C. Rouze, M. L. Palmeri, and K. R. Nightingale, "Ultrasonic shear wave elasticity imaging sequencing and data processing using a verasonics research scanner," IEEE Transactions on Ultrasonics, Ferroelectrics, and Frequency Control, vol. 64, no. 1, pp. 164-176, 2017.

[54] F. A. Duck, Physical Properties of Tissue: A comprehensive Reference Book. London: London: Academic Press, 1991.

[55] C. Kasai, K. Namekawa, A. Koyano, and R. Omoto, "Real-Time TwoDimensional Blood Flow Imaging Using an Autocorrelation Technique," IEEE Transactions on Sonics and Ultrasonics, vol. 32, no. 3, pp. 458464, 1985

[56] N. C. Rouze, M. H. Wang, M. L. Palmeri, and K. R. Nightingale, "Robust estimation of time-of-flight shear wave speed using a Radon sum transformation," Proceedings - IEEE Ultrasonics Symposium, vol. 57, no. 12 , pp. 21-24, 2010.

[57] J. A. Jensen and N. B. Svendsen, "Calculation of pressure fields from arbitrarily shaped, apodized, and excited ultrasound transducers," Ultrasonics, Ferroelectrics and Frequency Control, IEEE Transactions on, vol. 39, no. 2, pp. 262-267, 1992.

[58] L. Lacourpaille, F. Hug, K. Bouillard, J. Y. Hogrel, and A. Nordez, "Supersonic shear imaging provides a reliable measurement of resting muscle shear elastic modulus," Physiological Measurement, vol. 33, no. 3, 2012.

[59] H. Botanlioglu et al., "Shear wave elastography properties of vastus lateralis and vastus medialis obliquus muscles in normal subjects and female patients with patellofemoral pain syndrome," Skeletal Radiology, vol. 42, no. 5, pp. 659-666, 2013.

[60] M. L. Palmeri, A. Milkowski, R. Barr, P. Carson, M. Couade, J. Chen, S. Chen, M. Dhyani, R. Ehman, B. Garra, A. Gee, G. Guenette, Z. Hah, T. Lynch, M. Macdonald, R. Managuli, V. Miette, K. R. Nightingale, N. Obuchowski, N. C. Rouze, D. C. Morris, S. Fielding, Y. Deng, D. Chan, K. Choudhury, S. Yang, A. E. Samir, V. Shamdasani, M. Urban, K. Wear, H. Xie, A. Ozturk, B. Qiang, P. Song, S. McAleavey, S. Rosenzweig, M. Wang, Y. Okamura, G. McLaughlin, Y. Chen, D. Napolitano, L. Carlson, T. Erpelding, and T. J. Hall, "Radiological Society of North America/Quantitative Imaging Biomarker Alliance Shear Wave Speed
Bias Quantification in Elastic and Viscoelastic Phantoms," Journal of Ultrasound in Medicine, vol. 40, no. 3, pp. 569-581, 2021.

[61] C. A. Trutna, A. E. Knight, N. C. Rouze, L. D. Hobson-Webb, M. L. Palmeri, and K. R. Nightingale, "Viscoelastic Characterization in Muscle using Group Speed Analysis and Volumetric Shear Wave Elasticity Imaging," IEEE International Ultrasonics Symposium, IUS, vol. 2020September, no. 1, 2020.

[62] G. Y. Li, Q. He, L. X. Qian, H. Geng, Y. Liu, X. Y. Yang, J. Luo, and Y. Cao, "Elastic Cherenkov effects in transversely isotropic soft materials-II: Ex vivo and in vivo experiments," Journal of the Mechanics and Physics of Solids, vol. 94, pp. 181-190, 2016. [Online]. Available: http://dx.doi.org/10.1016/j.jmps.2016.04.028

[63] T. K. Yasar, T. J. Royston, and R. L. Magin, "Wideband MR elastography for viscoelasticity model identification," Magnetic Resonance in Medicine, vol. 70, no. 2, pp. 1-22, 2013. 